Reprod. Nutr. Dévelop., 1983, 23 (1), 101-114.

\title{
Identification et dosage des acides monomères à structure cyclique hydrogénés dans les lipides hépatiques du Rat ayant ingéré de l'huile de lin chauffée
}

\author{
B. POTTEAU, P. DUBOIS $(*), J . R I G A U D(*)$
}

Station de Recherches sur la Qualité des Aliments de I'Homme. (*) Laboratoire de Recherches sur les Arômes,

I.N.R.A., 17, rue Sully, BV 1540, 21034 Dijon Cedex, France.

Summary. Identification and quantitative analysis of hydrogenated cyclic monomeric acids in the hepatic lipids of rats ingesting heated linseed oil.

In a short-term (15 days) experiment, weanling Wistar rats were given diets at weaning containing $10 \%$ of different linseed oils : fresh linseed oil (lot F), linseed oil heatpolymerized at $275^{\circ} \mathrm{C}$ for $12 \mathrm{~h}$ under nitrogen (lot T) and linseed oil oxidized in the presence of air at $200^{\circ} \mathrm{C}$ for $100 \mathrm{~h}$ (lot $\mathrm{O}$ ).

We analyzed the fatty acids of total liver lipids and assayed the hydrogenated cyclic monomeric acids and identified them by using a combination of gas-liquid chromatography and mass spectrometry. Compared to lot $F$ animals, those of lots $T$ and $O$ showed considerable hepatic hypertrophy but total liver lipids were not increased. However, the usual fatty acid composition of these lipids was modified : the level of several monounsaturated (16:1, $18: 1$ ) and polyunsaturated (mainly $20: 4 n-6)$ fatty acids was increased in lots $T$ and $O$, which could result in increased activity of the enzymatic systems (desaturases) biosynthesizing them. Moreover, cyclic monomeric acids from heated oils were deposited at a 10 times higher concentration in the liver of lot $\mathrm{T}$ animals than in that of lot $\mathrm{O}$ animals...

The significance of these changes has been discussed.

\section{Introduction.}

Dans un travail antérieur (Potteau, Dubois et Rigaud, 1978), nous avons cherché à doser et à identifier les acides monomères à structure cyclique hydrogénés obtenus à partir de trois huiles de lin : huile de lin fraîche, huile de lin thermo-polymérisée à $275^{\circ} \mathrm{C}$ et huile de lin oxydée thermiquement à $200^{\circ} \mathrm{C}$.

L'objet principal du présent travail est de chercher à doser et à identifier les acides gras usuels et les acides monomères à structure cyclique dans le foie des rats ingérant ces huiles de lin chauffées.

Les méthodes d'analyse sont, en général, celles décrites dans le mémoire antérieur (Potteau, Dubois et Rigaud, 1978) ; dans certains cas nous avons procédé à des modifications qui seront précisées. 


\section{Matériel et méthodes.}

A. Essais sur animaux. - Vingt-sept rats mâles Wistar EOPS (*) provenant de l'élevage de la Station, âgés de 3 semaines, d'un poids moyen de $43,5 \mathrm{~g}$, reçoivent pendant une durée de 15 jours un régime contenant $10 \mathrm{p} .100$ d'huile de lin fraîche ou chauffée. La composition des constituants non lipidiques du régime est précisée dans un travail antérieur (Lhuissier et Potteau, 1971).

Trois lots expérimentaux de 9 rats sont constitués d'après la nature des lipides du régime:

- lot 1: régime à 10 p. 100 d'huile de lin fraiche iconsommation alignée sur celle des animaux du iot (II);

- lot 11 : régime à $10 \mathrm{p} .100 \mathrm{~d}^{\prime}$ hulle de $\operatorname{lin}$ chauffée à $275^{\circ} \mathrm{C}$ sous azote, pendant $12 \mathrm{~h}$;

- lot III : régime à 10 p. 100 d'huile de lin chauffée à $200^{\circ} \mathrm{C}$, à l'air pendant $100 \mathrm{~h}$.

B. Méthodes d'analyses. - A la fin des essais, les arimaux sont sacrifiés, les foies sont pesés puis congelés en attendant les analyses. Les lipides hépatiques sont extraits comme indiqué par Folch, Lees et Sloane-Stariley (1957) à partir des foies de 3 rats mis en pool ( 3 pools par lot expérimenta!). La détermination de la composition en acides gras des lipides totaux est effectuée comme il a été indiqué antérieurement (Potteau, 1974).

La détermination de la teneur des acides gras hépatiques en acides monomères à structure cyclique est effectuée par une méthode permettant de réaliser des dosages sur de très faibles quantités d'esters méthyliques (50 $\mathrm{mg}$ ou moins) provenant de lipides corporels.

Les esters méthyliques hydrogénés (catalyseur : oxyde de platine) sont fractionnés par dilution en deux parties :

- La première partie représente 4 p. 100 de l'échantillon initial. On y ajoute une quantité connue d'étalon interne (heptadecanoate de méthyle) et on déduit après chromatographie en phase gazeuse la quantité d'esters méthyliques usueis $18: 0$ à 24 : 0) de l'échantillon initial.

- La deuxième partie représente 96 p. 100 de l'échantillon de départ. On la dis-

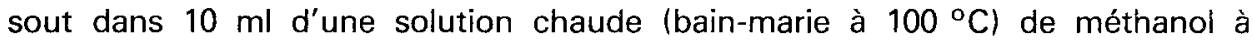
25 p. 100 d'urée, on agite, on laisse refroidir la solution à la température du laboratoire. Les cristaux d'urée formés sont séparés par filtration sur un verre fritté de porosité $n^{\circ} 2$ en s'aidant du vide, puis ils sont lavés trois fois avec $10 \mathrm{ml}$ de méthanol saturé en urée à la température du laboratoire. Le filtrat est transvasé dans une ampoule à décantation contenant $25 \mathrm{ml}$ d'eau distillée. On ajoute $2,5 \mathrm{ml} \mathrm{d} \mathrm{d}^{\prime} \mathrm{HCl} 6 \mathrm{~N}$, une quantité connue d'un étalon interne (heptadécanoate de méthyle) et $25 \mathrm{ml}$ d'un mélange d'éther de pétrole et d'éther éthylique $(1 / 1$, V/V). Après agitation et décantation, on sépare la phase éthérée qui est lavée à l'eau distillée, séchée sur sulfate de sodium et concentrée à l'évaporateur rotatif sous vide. L'échantillon est analysé par chromatographie en phase gazeuse sur

(*) EOPS = Exempts d'Organismes Pathogènes Spécifiques. 
une colonne contenant un support imprégné avec une phase polaire (succinate de butanedio'). La quantité de monomères cycliques est déterminée en calcuiant d'une part ia surface (à l'aide d'un planimètrel des pics situés entre 18 : 0 et 20 : 0 ipics $n^{c} 2$ à 8 ;, et d'autre part la surface du composé étaion.

Pour étudier la répétabilité des dosages d'acides monomères à structure cyclique, des échantilions de foie et de lait ont été analysés en 6 exemplaires. L'estimation de l'écart-type de chacune de ces séries de mesures a été basée, soit sur l'étendue, soit sur l'écart-type empirique (Snedecor et Cochran, 1957), et permet de conclure que l'erreur aléatoire commise sur le dosage des acides monomères à structure cyclique par cetie méthode est d'environ \pm 6 p. 100 .

L'identification des acides monomères à structure cycliquee a été effectuée sur des échantilions de lipides hépatiques de très jeunes ratons cont les mères ingéraient de I'huile de lin thermopolymérisée à $275^{\circ} \mathrm{C}$ (Potteau, 1976a). Les méthodes d'identification par couplage chromatographie en phase gazeuse spectrométrie de masse sont les mêmes que celles décrites dans notre travail antérieur (Potteau, Dubois et Rigaud, 1978). Un exemple de chromatogramme est montré sur la figure 1.

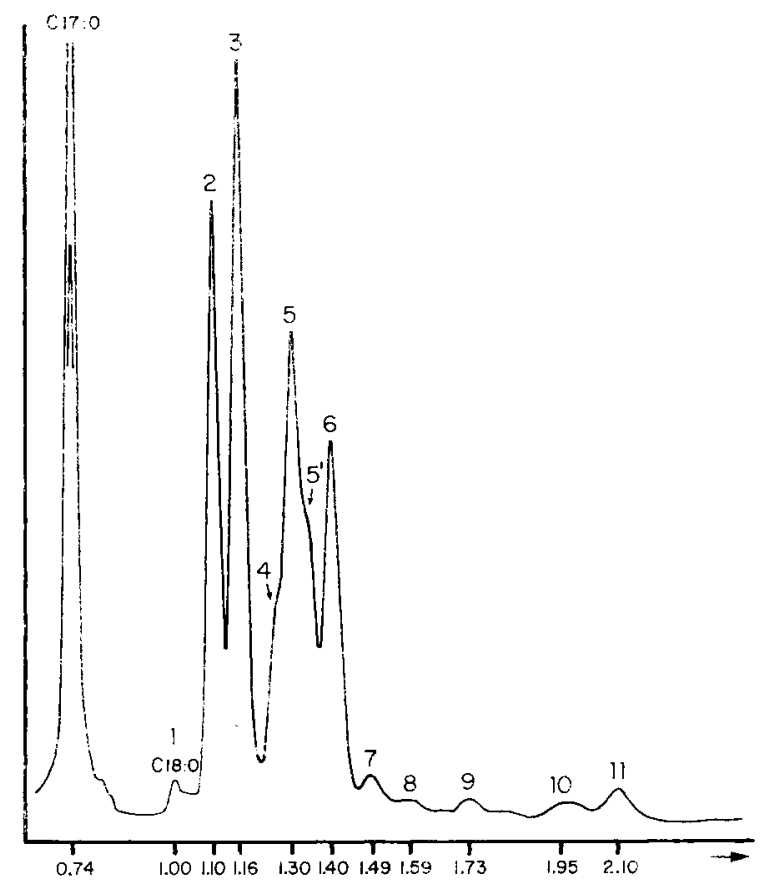

FIG. 1. - Chromatogramme obtenu à partir des esters méthyliques des lipides totaux du foie de jeunes ratons morts avant l'âge de 14 jours (Potteau, 1976a). Ces esters ont été hydrogénés, puis traités à l'urée. La chromatographie en phase gazeuse a été effectuée en utilisant du succinate de butane diol comme phase stationnaire, dans des conditions strictement comparabies à celles décrites dans notre mémoire antérieur (Potteau, Dubois et Rigaud, 1978). On a porté en abscisse le temps de rétention relatif $\alpha$ (par rapport au stéarate de méthyie). 


\section{Résultats.}

Après 15 jours d'expérience, les huiles chauffées n'ont pas d'effet significatif sur la taille du corps.

Cependant, on constate sur le tableau 1 que la taille du foie des animaux recevant les huiles chauffées est augmentée d'environ 80 p. 100 par rapport à celle du foie des témoins. II n'y a pas de différence significative entre les 2 lots recevant les huiles chauffées si on compare le poids du foie exprimé en g. Par contre si on calcule le poids du foie en p. $100 \mathrm{du}$ poids corporel, les animaux recevant l'huile thermopolymérisée présentent une taille relative du foie supérieure d'environ 10 p. 100 à celle des animaux recevant I'huile chauffée à l'air. Une analyse de covariance montre que cette différence est significative (au seuil de $p<0,01)$. Par contre, on ne relève pas de différence marquée entre les lots en ce qui concerne la taille d'autres organes (reins, cœur, cerveau, gonades).

Les teneurs en lipides totaux du foie des animaux des 3 lots expérimentaux sont normales; on ne relève pas de stéatose hépatique (tabl. 2). Par contre, la composition en acides gras des lipides totaux du foie est modifiée chez les animaux recevant les huiles chauffées. On trouve des teneurs augmentées en acides monoéniques (16: 1 et $18: 1$ ) et en acide triénique (20:3n - 9). On constate que les teneurs en acide linoléique sont diminuées, alors que les teneurs en certains métabolites de cet acide gras $(20: 3 n-6 ; 20: 4 n-6)$, en particulier celle de l'acide arachidonique, sont nettement augmentées chez les rats ingérant les huiles chauffées.

On constate également que les teneurs en acide linolénique sont nettement diminuées, ainsi que les teneurs de la plupart des métabolites de cet acide gras (20:5 $\mathrm{n}-3 ; 22: 5 \mathrm{n}-3$ ) à l'exception de l'acide docosahexaénoïque (22:6 $\mathrm{n}-3)$ qui ne présente pas de variation significative parmi les lots.

TABLEAU 1

Poids du corps et poids du foie.

\begin{tabular}{|c|c|c|c|}
\hline & $\begin{array}{l}\text { Lot } 1 \\
\text { Huile fraîche }\end{array}$ & $\begin{array}{c}\text { Lot II } \\
\text { Huile } \\
\text { thermopolymérisée } \\
\text { à } 275^{\circ} \mathrm{C}\end{array}$ & $\begin{array}{c}\text { Lot III } \\
\text { Huile } \\
\text { chauffée à l'air } \\
\text { à } 200^{\circ} \mathrm{C}\end{array}$ \\
\hline Poids du corps en g (a) & $96,7 \pm 4,74$ & $93,0 \pm 5,44$ & $100,5 \pm 1,96$ \\
\hline Poids du foie en $\mathrm{g}(\mathrm{a})$ & $\frac{4,21}{(2)} \pm 0,320$ & $7,56 \underset{\left(\frac{1}{1}\right)}{0,429}$ & $7,51_{(1)}^{ \pm} 0,198$ \\
\hline $\begin{array}{l}\text { Poids du foie en p. } 100 \\
\text { du poids du corps }(a)\end{array}$ & $4,30 \pm 0,154$ & $8,13 \pm 0,075$ & $7,47 \pm 0,116$ \\
\hline Poids du foie ajusté (b) & $\underset{(2)}{4,21} \pm \underset{(3)}{0,098}$ & $\begin{array}{c}7,83 \pm 0,098 \\
(1) \quad(3)\end{array}$ & $\underset{(1)}{7,23} \pm \underset{(2)}{0,098}$ \\
\hline
\end{tabular}

(a) Moyenne de 9 déterminations \pm écart type de la moyenne.

(b) Les résultats proviennent d'une analyse statistique de la covariance.

(1) Différence significative avec le lot I au seuil de $P<0,01$.

(2) Différence significative avec le lot II au seuil de $P<0,01$.

(3) Différence significative avec le lot III au seuil de $P<0,01$. 
TABLEAU 2

Composition en acides gras usuels et en acides monomères à structure cyclique dans les lipides totaux du foie.

\begin{tabular}{cccc}
\hline & $\begin{array}{c}\text { Lot I } \\
\text { Huile fraîche }\end{array}$ & $\begin{array}{c}\text { Lot II } \\
\text { Huile chauffée } \\
\text { à } 275^{\circ} \mathrm{C}\end{array}$ & $\begin{array}{c}\text { Lot III } \\
\text { Huile chauffée } \\
\text { à } 200^{\circ} \mathrm{C}\end{array}$ \\
\hline $\begin{array}{c}\text { Lipides totaux du foie } \\
\text { (p. 100) (a) }\end{array}$ & $\begin{array}{c}4,70 \\
(4,46-4,82-4,81)\end{array}$ & $\begin{array}{c}4,99 \\
(4,87-4,85-5,25)\end{array}$ & $(4,60-4,12-4,33)$ \\
\hline
\end{tabular}

Composition en acide gras usuels (p. 100) (a)

\begin{tabular}{|c|c|c|c|}
\hline $14: 0$ & $\begin{array}{l}0,34 \\
(0,43-0,39-0,19)\end{array}$ & $\begin{array}{c}0,40 \\
(0,46-0,42-0,31)\end{array}$ & 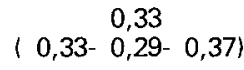 \\
\hline $16: 0$ & $\begin{array}{c}21,85 \\
(20,57-21,61-23,36)\end{array}$ & $\begin{array}{c}22,93 \\
(24,69-22,52-21,59)\end{array}$ & $\begin{array}{c}25,38 \\
(25,06-25,27-25,81)\end{array}$ \\
\hline $16: 1 n-7$ & $\begin{array}{c}2,45 \\
(2,62-2,48-2,24)\end{array}$ & $\begin{array}{l}2,85 \\
(3,09-3,10-2,37)\end{array}$ & $\begin{array}{l}3,46 \\
(3,76-2,96-3,65)\end{array}$ \\
\hline $18: 0$ & $\begin{array}{c}16,24 \\
(15,20-16,52-16,99)\end{array}$ & $\begin{array}{c}13,13 \\
(12,38-13,15-13,87)\end{array}$ & $\begin{array}{c}12,97 \\
(11,38-14,04-13,48)\end{array}$ \\
\hline $18: 1 n-9$ & $\begin{array}{c}14,08 \\
(15,33-13,91-13,01)\end{array}$ & $\begin{array}{c}18,29 \\
(19,17-18,26-17,45)\end{array}$ & $\begin{array}{c}20,53 \\
(22,86-19,46-19,27)\end{array}$ \\
\hline $18: 2 n-6$ & $\begin{array}{c}12,12 \\
(12,15-11,45-12,76)\end{array}$ & $\begin{array}{c}10,83 \\
(10,30-10,46-11,73)\end{array}$ & $\begin{array}{c}8,52 \\
(8,09-8,77-8,70)\end{array}$ \\
\hline $18: 3 n-3$ & $\begin{array}{c}7,54 \\
(8,79-7,64-6,20)\end{array}$ & $\begin{array}{c}4,17(b) \\
(3,95-4,04-4,52)\end{array}$ & $\begin{array}{c}2,80(b) \\
(2,77-3,12-2,52)\end{array}$ \\
\hline $20: 3 n-9$ & $\begin{array}{l}0,12 \\
(0,08 \cdot-0,17-0,12)\end{array}$ & $\begin{array}{l}0,84 \\
(0,69-0,97-0,87)\end{array}$ & $\begin{array}{c}0,54 \\
(0,61-0,50-0,52)\end{array}$ \\
\hline $20: 3 n-6$ & $\begin{array}{ll} & 0,66 \\
(0,70- & 0,67-0,60)\end{array}$ & $\begin{array}{c}1,01 \\
(1,00-1,10-0,93)\end{array}$ & $\begin{array}{l}1,10 \\
(1,32-1,12-0,85)\end{array}$ \\
\hline $20: 4 n-6$ & $\begin{array}{c}6,90 \\
(6,00-6,38-8,32)\end{array}$ & $\begin{array}{c}13,93 \\
(12,64-14,11-15,03)\end{array}$ & $\begin{array}{c}9,71 \\
(9,30-9,76-10,08)\end{array}$ \\
\hline $20: 5 n-3$ & $\begin{array}{c}7,53 \\
(8,22-8,12-6,26)\end{array}$ & $\begin{array}{c}2,40(b) \\
(2,41-2,57-2,23)\end{array}$ & $\begin{array}{ll} & 5,11(b) \\
(4,79-5,65-4,90)\end{array}$ \\
\hline $22: 5 n-3$ & $\begin{array}{l}2,45 \\
(2,41-2,73-2,20)\end{array}$ & $\begin{array}{l}1,07 \\
(1,16-1,05-0,99)\end{array}$ & $\begin{array}{c}2,34 \\
(2,31-2,48-2,22)\end{array}$ \\
\hline $22: 6 n-3$ & $\begin{array}{c}6,30 \\
(5,93-6,52-6,44)\end{array}$ & $\begin{array}{l}\quad 6,27 \\
(6,18-6,21-6,43)\end{array}$ & $\begin{array}{l}5,90 \\
(5,73-5,82-6,16)\end{array}$ \\
\hline$=0$ & $\begin{array}{c}0,010 \\
(0,011-0,012-0,008)\end{array}$ & $\begin{array}{c}1,36 \\
(1,55-1,37-1,16)\end{array}$ & $\begin{array}{c}0,150 \\
(0,156-0,151-0,144)\end{array}$ \\
\hline
\end{tabular}

(a) Moyenne de 3 déterminations.

(b) Valeur par excès, le pic obtenu par chromatographie en phase gazeuse correspondant manifestement à la présence de plusieurs composés mal séparés.

Enfin, on note la présence d'acides monomères à structure cyclique chez les animaux recevant les deux types d'huiles chauffées. La teneur en ces composés est beaucoup plus élevée chez les animaux recevant l'huile thermopolymérisée $(1,36$ p. 100) que chez ceux recevant l'huile chauffée à l'air $(0,15$ p. 100). Chez les témoins recevant l'huile fraîche, ces composés ne sont présents qu'à l'état de traces $(0,01$ p. 100). 
Les différents pics correspondant à des acides monomères à structure cyclique qui ont pu être séparés par chromatograpịie en phase gazeuse ont été caractérisés par leur temps de rétention relatif, et dans certains cas par couplage chromatographie en phase gazeuse - spectrométrie de masse.

Les temps de rétention relatifs qui sont donnés dans le tableau 3 pour ces composés cycliques (pics $n^{\circ} 2$ à 11 ) ont été cléterminés lors d'une chromatographie effectuée dans des conditions icientiques à celles utilisées lors de notre travai! antérieur (Potteau, Dubois et Rigaud, 1978). Les pourcentages approximatifs en ces différents composés sont également donnés sur le tableau 3. Les spectres de masse obtenus par couplage CPG - SM pour los pics $n^{\circ} 2,3,5,\left(+4+5^{\prime}\right)$, 6 et 7 confirmert ce que nous avions observé dans notre travail antérieur (Potteau, Dubois et Pigaud, 1978):

- Les pics 4, 5 et 5 ' (mal séparés), le pic 6, le pic 7 correspondent à des dérivés à structure cyclohexane 1,2 - disubstitués: $\mathrm{H}\left(\mathrm{CH}_{2}\right\rangle_{n}-\mathrm{C}_{6} \mathrm{H}_{10}-\left(\mathrm{CH}_{2}\right)_{11}-\mathrm{n}-$ $\mathrm{COO} \mathrm{CH}_{3}$ avec $n=2,3$ et 4 , les composés pour lesqueis $n=3$ (pics $n^{0} 5$ et 5 ) étant les plus abondants.

- Le pic 2 et le pic 3 pourraient correspondre principalement à des dérivés à structure cyclopentane 1,2 - disubstitués: $\mathrm{H}\left(\mathrm{CH}_{2}\right)_{n}-\mathrm{C}_{5} \mathrm{H}_{3}-\left(\mathrm{CH}_{2}\right)_{12}-\mathrm{n}$ COO $\mathrm{CH}_{3}$ avec $n=3$ (pic 3) et $n=4$ (pic 2).

Cependant, il est possible que la plupart de ces pics ne correspondent pas à des composés purifiés, les structures proposées correspondant vraisemblablement aux composés prédominants pour chacun de ces pics. Nous renvoyons à notre travail antérieur (Potteau. Dubois et Rigaud, 1978) où nous avons donné les arguments nous permettant d'étayer les hypothèses précédentes concernant les structures proposées. En ce qui concerne les teneurs approximatives en ces différents constituants (tabl. 3), on remarque que les pics $n^{\circ} 2$ et 3 , qui sont constitués pour l'essentiel par des acides monomères à structure cyclopentanique, représentent 33 p. 100 des acides monomères à structure cyclique hydrogénés obtenus à partir de l'huile de lin thermopolymérisée.

Dans le foie des rats sevrés ingérant cette huile thermopolymérisée, au terme de l'expérience de 15 jours décrite dans le présent travail, ces composés à structure cylopentanique sont présents à des concentrations de l'ordre de $40 \mathrm{p}$. 100.

Dans le lait de ratte en lactation ingérant cette huile thermopolymérisée (Potteau, 1976b), ces composés sont également présents à des concentrations de l'ordre de 40 p. 100.

Dans le foie des jeunes ratons morts (Potteau, 1976a) ou sacrifiés (Potteau, 1976b) avant l'âge du sevrage, qui ont été élevés par des rattes recevant l'huile thermopolymérisée, la teneur en ces composés est encore plus élevée : de l'ordre de 45 p. 100. On peut remarquer que ces augmentations de teneur, dans les lipides du foie, sont principalement attribuables au pic $n^{\circ} 2$, qui correspond essentiellement à un ester cyclopentanique à substituant butyle.

Par contre, on constate dans les lipides du foie, une forte diminution de la teneur du pic $n^{\circ} 6$ qui correspond essentiellement à I'un des deux isomères de configuration, correspondant eux-mêmes aux esters cyclohexaniques à substituant propyle : cet isomère est présent à une concentration de l'ordre de $24 \mathrm{p}$. 


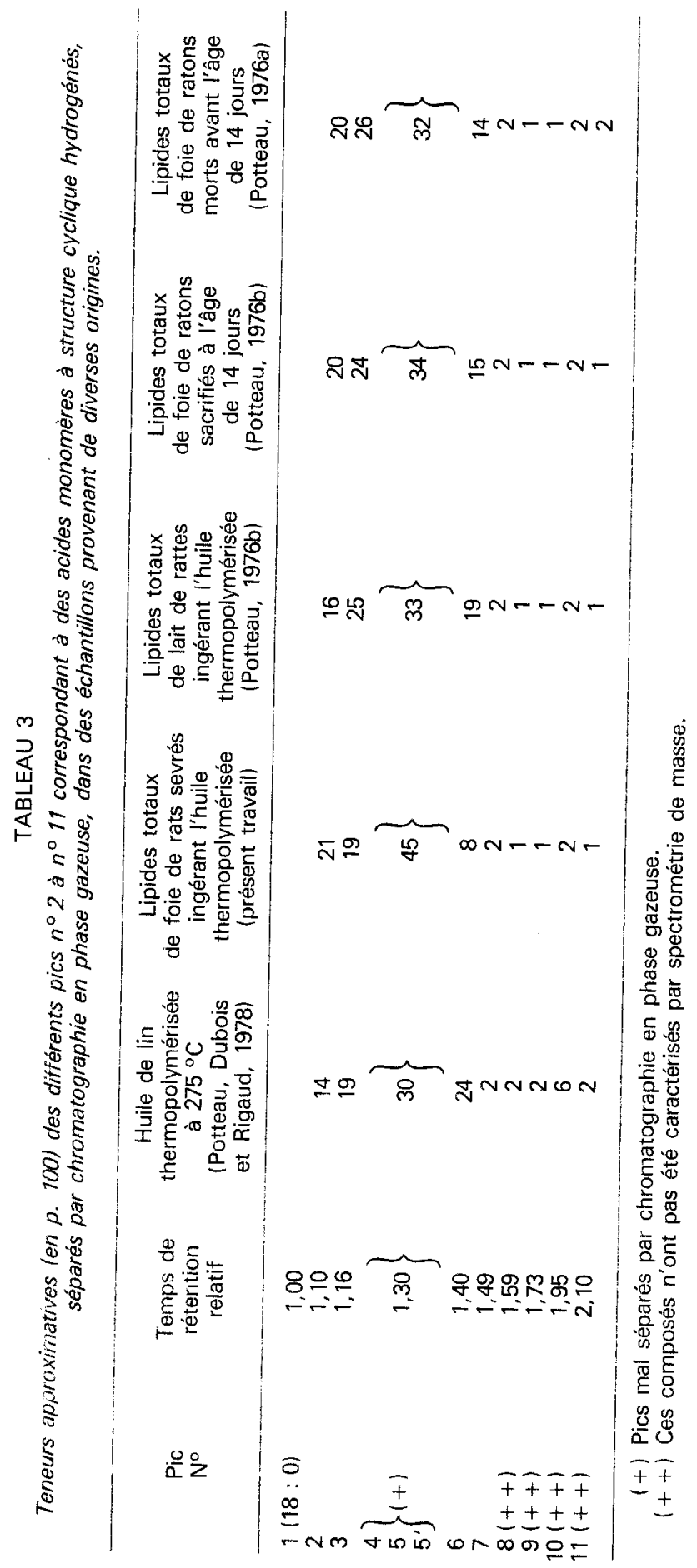


100 dans I'huile de lin thermopolymérisée, alors que dans le foie des rats sevrés ayant ingéré cette huile pendant 15 jours, sa concentration n'est plus que de l'ordre de 8 p. 100.

On peut penser que, parmi les différents composés cycliques ingérés, ceux qui, comme pour le pic $n^{\circ} 6$, ont des teneurs diminuées dans les lipides du foie, sont ceux que l'organisme parvient à cataboliser ou à éliminer le plus rapidement ; alors qu'au contraire, ceux qui, comme pour le pic $n^{\circ} 2$, ont des teneurs augmentées dans les lipides du foie, sont ceux que l'organisme catabolise ou élimine le plus lentement.

\section{Discussion et conclusion.}

Dans le présent travail, nous avons administré à de jeunes rats à l'âge du sevrage, des huiles de lin fraîche et chauffées pendant une durée expérimentale courte (15 jours). Dans ces conditions, nous avons obtenu avec l'huile thermopolymérisée une hypertrophie hépatique beaucoup plus marquée laugmentation de l'ordre de 80 p. 100 par rapport aux témoins) que celle relevée après une durée expérimentale de 2 à 3 mois laugmentation de l'ordre de 20 p. 100 par rapport aux témoins (Grandgirard, Potteau et Mitjavila, 1972). Cette hypertrophie hépatique très marquée est à rapprocher du fait que Lhuissier et Potteau (1971) ont constaté, chez des rats recevant des huiles de soja et de lin thermopolymérisées, un abaissement du rejet urinaire de certaines vitamines coenzymatiques du groupe $\mathrm{B}$, conjointement avec une augmentation de la teneur hépatique des mêmes vitamines. Ces résultats ont conduit à formuler l'hypothèse de la formation, au cours du chauffage des huiles, de composés provoquant une induction du système enzymatique au niveau du foie. Les composés responsables de ces effets sont présents dans la fraction où sont concentrés les acides monomères à structure cyclique (Lhuissier et Potteau, 1972). Cette hypothèse d'une induction enzymatique au niveau du foie de rats recevant des corps gras chauffés a ultérieurement été reprise et confirmée par Lang (1973) pour expliquer une durée de sommeil plus courte après une administration d'hexobarbital, par Grandgirard et Loisel (1974) pour expliquer une augmentation d'activité de la phosphatase alcaline du sérum, et par Andia et Street (1975) pour expliquer une augmentation de la teneur en protéines microsomales et une augmentation d'activité de plusieurs systèmes enzymatiques du foie.

Rao, Hemans et Perkins (1973) ont chauffé de l'huile de maïs en présence d'air à $190{ }^{\circ} \mathrm{C}$ pendant $132 \mathrm{~h}$ et ont administré cette huile à de jeunes rats sevrés pendant 18 semaines. Ils ont constaté une augmentation de la taille du foie, ainsi qu'une nette augmentation de sa teneur en lipides totaux. A la différence de ces auteurs, nous n'avons pas observé de stéatose hépatique chez le Rat sevré, ni dans le cadre de la présente expérience à court terme (15 jours), ni dans une expérience antérieure à moyen terme (2 à 3 mois) (Potteau, 1974). Dans nos conditions de travail, nous n'avons pu mettre en évidence une stéatose hépatique que dans des conditions particulières d'administration, par intubation stomacale chez le Rat sevré (Grandgirard, Nouvelot et Potteau, 1972 ; Potteau et 
Grandgirard, 1974), ou encore dans le cadre d'expériences effectuées sur de très jeunes ratons avant l'âge du sevrage (Potteau, 1976a et b; Potteau et al., 1977).

Rao, Hemans et Perkins (1973) ont administré par injection intrapéritonéale de l'acétate de sodium $1^{14} \mathrm{C}$ aux animaux peu de temps avant le sacrifice. Ils constatent que le $1^{14} \mathrm{CO}_{2}$ est expiré plus lentement chez les animaux traités recevant I'huile chauffée par rapport aux témoins, ce qui indique que l'acétate $1^{14} \mathrm{C}$ est retenu dans l'organisme pour une utilisation préférentielle en vue de la synthèse des lipides. Par ailleurs, l'analyse des acides gras totaux du foie séparés par chromatographie sur couche mince en classes selon leur degré d'insaturation, révèle qu'il $y$ a une incorporation fortement augmentée (environ doublée), par rapport aux témoins, de l'activité ${ }^{14} \mathrm{C}$ provenant de l'acétate dans toutes les classes $d$ 'acides gras insaturés : monoènes, diènes, triènes et tétraènes. Selon les auteurs, cette observation pourrait indiquer un effet de stimulation (induction enzymatique) des systèmes enzymatiques responsables de la synthèse des acides gras insaturés. Bien que ces acides gras n'aient pas été identifiés, on peut penser qu'il s'agit: pour les monoènes, essentiellement des acides $16: 1 \mathrm{n}-7$ et $18: 1 n-9$ pour les triènes et tétraènes, des $20: 3 n-9,20: 3 n-6$ et $20: 4 n-6$ (acide arachidonique). Dans un travail antérieur (Potteau, 1974) et dans le présent travail, nous observons une augmentation de la teneur de ces acides gras, ce qui confirmerait l'hypothèse que nous avions formulée (Potteau, 1974) pour le seul cas de l'acide $16: 1 \mathrm{n}-7$ et celle de Rao, Hemans et Perkins (1973) pour l'ensemble des acides insaturés des monoènes aux tétraènes.

Rao, Hemans et Perkins (1973) indiquent d'autre part que l'augmentation de I'incorporation de la radioactivité ${ }^{14} \mathrm{C}$ a lieu essentiellement au niveau de la classe des triglycérides. Ce résultat est à rapprocher des travaux de Gabriel, Alexander et Valli (1977, 1978), qui ont préparé des fractions (obtenues par distillation des esters éthyliques à $150-180^{\circ} \mathrm{C}$ ) provenant de différentes huiles chauffées à $180{ }^{\circ} \mathrm{C}$ pendant $72 \mathrm{~h}$ à l'air et les ont administrées à de jeunes rats sevrés pendant 28 jours. Ces auteurs montrent que les effets de ces fractions sur la composition en acides gras de lipides tissulaires sont très différents, suivant qu'il s'agit de lipides neutres ou de lipides polaires. C'est ainsi que les teneurs en acide arachidonique sont fortement élevées dans les lipides neutres du foie, mais cet effet ne se retrouve pas au niveau des lipides polaires.

L'examen de cet ensemble de résultats appelle plusieurs interrogations parmi lesquelles nous en retiendrons deux.

1) La première interrogation porte sur la comparaison des effets respectifs des deux huiles de lin chauffées au niveau du foie.

L'hypertrophie hépatique observée chez les rats ayant reçu l'huile oxydée à $200{ }^{\circ} \mathrm{C}$ est presque équivalente à celle observée chez les rats ayant reçu l'huile thermopolymérisée à $275^{\circ} \mathrm{C}$, alors que les quantités de monomères cycliques ingérés par rat au cours des 15 jours sont respectivement de $146 \mathrm{mg}$ et $1351 \mathrm{mg}$ pour ces deux lots, c'est-à-dire dans un rapport de l'ordre de 1: 10. Bien que nous $n^{\prime}$ ayons pas mis en évidence de stéatose hépatique, les lipides du foie des rats recevant ces deux huiles chauffées contiennent des acides monomères à 
structure cyciique à des concentrations (respectivement 0,15 et 1,36 p. 100) qui sont à peu près dans le même rapport $1: 10$, que celui des quantités de ces composés ingérées par les animaux. Cependant, le travail de Friedman et al. (1961) avait établi qu'il existait une régression linéaire entre le logarithme du rapport poids du foie/poids du corps et le logarithme de la dose introduite dans le régime de la fraction contenant les acides ne formant pas de complexe d'inclusion avec l'urée (où se trouvent concentrés les acides monomères à structure cyclique) isolée à partir d'une huile de coton oxydée à $225^{\circ} \mathrm{C}$. La responsabilité des acides monomères à structure cyclique dans le développement d'une hypertrophie hépatique a ultérieurement été confirmée en comparant l'effet de fractions préparées à partir d'une huile de soja thermopolymérisée (Potteau et al., 1970) ou d'acide linolénique purifié chauffé en présence de base forte (lwaoka et Perkins, 1976), les polymères de type thermique ne provoquant pas cette anomalie (Potteau et al., 1970), Hsieh et Perkins, 1976).

On peut donc se demander pourquoi l'huile oxydée à $200^{\circ} \mathrm{C}$ provoque une hypertrophie hépatique pratiquement équivalente à celle obtenue avec l'huile thermopolymérisée à $275^{\circ} \mathrm{C}$, alors qu'elle contient 10 fois moins d'acides monomères à structure cyclique. Plusieurs éventualités peuvent être envisagées pour I'expliquer.

Les monomères à structure cyclique formés lors de traitements oxydatifs à $200{ }^{\circ} \mathrm{C}$ ont-ils les mêmes structures que ceux formés au cours de la thermopolymérisation à $275^{\circ} \mathrm{C}$ ? Des différences, autres que celles portant sur la nature du cycle et sur la longueur respective des deux chaînes latérales, peuvent concerner le nombre, la position et la configuration des doubles liaisons. Des différences de structure pourraient contribuer à expliquer des différences d'effets physiologiques par exemple sur la toxicité ou sur l'hypertrophie du foie. Cette éventualité est suggérée par les résultats de travaux de Crampton et a/. (1953, 1956), Nolen, Alexander et Artman (1967), Artman (1969) qui ont montré que l'administration à de jeunes rats sevrés de doses équivalentes de fractions loù sont concentrés les acides monomères à structure cyclique) obtenues à partir de différents corps gras chauffés causent des effets toxiques pius ou moins prononcés. Un argument plus probant a récemment été apporté par Saito et Kaneda (1976) qui ont séparé, à partir des monomères cycliques provenant d'une huile de lin thermopolymérisée, plusieurs fractions qu'ils ont administrées à des souris. Certaines fractions se sont révélées beaucoup plus toxiques que d'autres à dose d'administration égale.

Dans !'huile de lin oxydée à $200^{\circ} \mathrm{C}$, ne se forme-t-il pas d'autres chaînes grasses atypiques qui n'ont pas été dosées et caractérisées dans le présent travail et qui pourraient contribuer à I'hypertrophie du foie ? Une hypertrophie hépatique a été observée par d'autres auteurs chez des rats ou des souris recevant des corps gras présentant une teneur élevée en acides gras monoéniques de configu ration trans (Raulin, 1960 ; Vles et Gottenbos, 1972 ; Astorg et Cluzan, 1976), en acides gras linéaires hydroxylés (Perkins, Endres et Kummerow, 1961) en acides gras époxydés (Kiechebusch et al., 1963), en polymères de type thermo-oxydatif (riches en fonctions hydroxylées, séparés à partir d'une huile de maïs oxydée à $20{ }^{\circ} \mathrm{C}$ ) (Perkins et Kummerow, 1959), ou en certains produits formés lors de 
I'autoxydation à des températures relativement peu élevées (de l'ambiante à $100^{\circ} \mathrm{C}$ ) de différents corps gras (Poling et al., 1962 ; Kaunitz et al., 1959 ; Nakamura et al., 1973).

La méthode que nous avons utilisée permet-elle de déceler et de doser tous les acides monomères à structure cyclique qui ont pu se former dans les huiles de lin chauffées ? En particulier, nous n'avons pas pu mettre en évidence la présence d'acides benzéniques, ni d'acides cycliques portant une fonction oxygénée (cétonique ou hydroxylée) du type de ceux isolés par Michael, Alexander et Artman (1966), Michael (1966), Artman et Alexander (1968), Artman et Smith (1972), à partir de corps gras riches en acide linoléique (mais ne contenant pas d'acide linolénique) oxydés à des températures de l'ordre de 180 à $200{ }^{\circ} \mathrm{C}$. Le métabolisme des acides benzéniques 1-2 disubstitués a été bien étudié (Gottenbos et Thomasson, 1965 ; Van Tilborg et al., 1970) ; ils sont responsables en particulier d'une nette augmentation de la taille du foie. Par contre, à notre connaissance, on na pas d'informations sur les possibilités de formation, les méthodes d'isolement et de caractérisation et l'éventuelle action physiologique des acides cycliques portant une fonction oxygénée, à part celles données dans le travail d'Artman et Smith (1972).

Ces éventualités n'ont pas été étudiées dans le présent travail et demanderaient des recherches complémentaires.

2) La deuxième interrogation porte sur la signification de l'augmentation de la teneur des lipides du foie en certains acides gras insaturés.

Dans le cas de l'augmentation de la teneur en acide palmitoléique dans les acides gras totaux du foie ou du tissu adipeux (Potteau, 1974), nous avions formulé I'hypothèse d'une augmentation de l'activité du système enzymatique effectuant la désaturation de l'acide palmitique. Actuellement, on peut faire l'hypothèse que d'autres systèmes enzymatiques permettant de faire la synthèse d'acides gras insaturés sont concernés. A la suite des travaux de Rao, Hemans et Perkins (1973), de Gabriel, Alexander et Valli $(1977,1978)$ et du présent travail, on peut penser essentiellement au système enzymatique permettant d'effectuer la biosynthèse de l'acide arachidonique à partir de l'acide linoléique, et peut-être aussi aux systèmes enzymatiques effectuant la biosynthèse de l'acide oléique (à partir de l'acide stéarique) et de l'acide $20: 3 n-9$ (à partir de l'acide oléique). Bien que les travaux précités ne le suggèrent pas, on peut également faire I'hypothèse que l'activité du système enzymatique effectuant la biosynthèse de l'acide docosahexanéoique (22:6n-3) à partir de l'acide linolénique, serait augmentée. En effet, dans un travail antérieur (Potteau, 1974) et dans le présent travail, il est frappant de constater que la teneur en $22: 6 n-3$ dans les lipides hépatiques des rats ingérant les huiles chauffées, reste stable par rapport aux témoins, alors qu'au contraire l'acide linolénique et la plupart des autres métabolites intermédiaires (20:5n-3,22:5n-3) ont des teneurs très nettement réduites. D'autres expériences, effectuées sur des rats beaucoup plus jeunes (avant l'âge du sevrage) (Potteau, 1976a, 1976b ; Potteau et al., 1977 ; Potteau, résultats non publiés), étayeraient cette dernière hypothèse, puisque, dans ces 
conditions, on observe dans les lots recevant les huiles chauffées une augmentation de la teneur en 22: 6 n -3 dans les lipides hépatiques des jeunes.

Des facteurs présents dans les huiles chauffées pourraient donc augmenter l'activité de la plupart des systèmes enzymatiques (et particulièrement des désaturases) permettant d'effectuer la synthèse d'acides gras insaturés au niveau du foie. Des travaux complémentaires seraient nécessaires pour étayer cette hypothèse et pour essayer de préciser à quels composés atypiques formés au cours du chauffage incombe la responsabilité de ces effets.

Reçu en décembre 1980.

Accepté en septembre 1982

\section{Références}

ANDIA A. M. G., STREET J. C., 1975. Dietary induction of hepatic microsomal enzymes by thermally oxidized fats. J. agric. Food Chem., 23, 173-177.

ARTMAN N. R., 1969. The chemical and biological properties of heated and oxidized fats. Adv. Lipid Res., 7, 245-330.

ARTMAN N. R., ALEXANDER J. C., 1968. Characterization of some heated fat components. J. am. Oil Chem. Soc., 45, 643-648.

ARTMAN N. R., SMITH D., 1972. Systematic isolation and identification of minor components in heated and unheated fat. J. am. Oil Chem. Soc., 49, 318-326.

ASTORG P. O., CLUZAN R., 1976. Effets physiologiques comparés d'huiles de hareng partiellement hydrogénées et de l'huile de colza chez le rat. I. Effets à moyen terme sur la croissance, le poids des organes et l'histologie du cœur. Ann. Nutr. Alim., 30, 581-602.

CRAMPTON E. W., COMMON R. H., FARMER F. A., WELLS A. F., CRAWFORD D., 1953. Studies to determine the nature of the damage to the nutritive value of some vegetable oils from heat treatment. III. The segregation of toxic and non-toxic material from the esters of heat-polymerized linseed oil by distillation and by urea adduct formation. J. Nutr., 49, 333346.

CRAMPTON E. W., COMMON R. H., PRITCHARD E. T., FARMER F. A., 1956. Studies to determine the nature of the damage to the nutritive value of some vegetable oils from heat treatment. IV. Ethyl esters of heat-polymerized linseed, soybean and sunflower seed oils. J. Nutr., 60, 13-24.

FOLCH J., LEES M., SLOANE-STANLEY G. H., 1957. A simple method for the isolation and purification of total lipids from animal tissues. J. biol. Chem., 226, 497-509.

FRIEDMAN L., HORWITZ W., SHUE G. M., FIRESTONE D., 1961. Heated fats. II. The nutritive properties of heated cottonseed oil and of heated cottonseed oil fractions obtained by distillation and urea adduct formation. J. Nutr., 73, 85-93.

GABRIEL H. G., ALEXANDER J. C., VALLI V. E., 1977. Biochemical and histological effects of feeding thermally oxidized rapeseed oil and lard to rats. Can J. compar. Med., 41, 98-106.

GABRIEL H. G., ALEXANDER J. C., VALLI V. E., 1978. Nutritional and metabolic studies of distillable fractions from fresh and thermally oxidized corn oil and olive oil. Lipids, 13, 49-55.

GOTTENBOS J. J., THOMASSON H. H., 1965. The biological action of hardened oils. Nutr. Diet., 7, 110-129.

GRANDGIRARD A., LOISEL W., 1974. Influence de I'ingestion d'huile de lin chauffée sur l'activité de la phosphatase alcaline et des transaminases sériques, chez le rat. Ann. Nutr. Alim., 28, 121-133.

GRANDGIRARD A., NOUVELOT A., POTTEAU B., 1972. Influence de l'ingestion par le rat d'huiles végétales thermopolymérisées sur quelques caractéristiques hépatiques. Bull. Groupe Lipides et Nutrition, $\mathrm{n}^{\circ} 4,34-39$.

GRANDGIRARD A., POTTEAU B., MITJAVILA S., 1972. Influence des huiles de soja et de lin thermopolymérisées sur l'activité de quelques systèmes enzymatiques au niveau du foie (chez le rat). Ann. Nutr. Alim., 26, 161-178. 
HSIEH A., PERKINS E. G., 1976. Nutrition and metabolic studies of methyl esters of dimeric fatty acids in the rat. Lipids, 11, 763-768.

IWAOKA W. T., PERKINS E. G., 1976. Nutritional effects of the cyclic monomers of methyl linolenate in the rat. Lipids, 11, 349-353.

KAUNITZ H., SLANETZ C. A., JOHNSON R. E., KNIGHT H. B., KOOS R. E., SWERN D., 1959. Influence of feeding fractionated esters of autoxidized lard and cottonseed oil on growth, thirst, organ weights, and liver lipids of rats. J. am. Oil Chem. Soc., 36, 611-615.

KIECKEBUSCH W., JAHR K., CZOK G., DEGKWITZ E., LANG K., 1963. Physiologische Eigenschaften epoxydierter Öle. Fette Seifen Anstrichm., 65, 919-924.

LANG K., 1973. Die physiologischen wirkungen erhitzter fette, insbesondere der frittierfette. Fette Seifen Anstrichm., 75, 73-76.

LHUISSIER M., POTTEAU B., 1971. Influence des huiles de soja et de lin thermopolymérisées sur la teneur du foie en thiamine, riboflavine, vitamine $B_{6}$ et niacine (chez le rat). Ann. Nutr. Alim., 25, 215-221.

LHUISSIER M., POTTEAU B., 1972. Influence de différentes fractions préparées à partir d'huile de soja chauffée sur le métabolisme de la thiamine et de la riboflavine (chez le rat). Ann. Biol. anim. Bioch. Biophys., 12, 335-339.

MICHAEL W. R., 1966. Thermal reactions of methyl linoleate. Il. The structure of aromatic $\mathrm{C}_{18}$ methyl esters. Lipids, 1, 359-364.

MICHAEL W. R., ALEXANDER J. C., ARTMAN N. R., 1966. Thermal reactions of methyl linoleate. I. Heating conditions, isolation technique, biological studies and chemical changes. Lipids, 1, 353-358.

NAKAMURA M., TANAKA H., HATTORI Y., WANATABE M., 1973. Biological effects of autoxidized safflower oils. Lipids, 8, 566-572.

NOLEN G. A., ALEXANDER J. C., ARTMAN N. R., 1967. Long-term rat feeding study with used frying fats. J. Nutr., 93, 337-348.

PERKINS E. G., ENDRES J. G., KUMMEROW F. A., 1961. Effect of ingested thermally oxidized corn oil on fat composition in the rat. Proc. Soc. exp. Biol. Med., 106, 370-372.

PERKINS E. G., KUMMEROW F. A., 1959. The nutritional effect of polymers isolated from thermally oxidized corn oil. J. Nutr., 68, 101-108.

POLING C. E., WARNER W. D., MONE P. E., RICE E. E., 1962. The influence of temperature, heating time, and aeration upon the nutritive value of fats. J. am. Oil Chem. Soc., 39, 315320.

POTTEAU B., 1974. Influence de l'ingestion d'huile de lin chauffée sur l'utilisation digestive et la composition en acides gras des lipides du foie et des dépôts adipeux chez le rat mâle en croissance. Ann. Nutr. Alim., 28, 135-158.

POTTEAU B., 1976a. Influence d'huiles de lin chauffées sur la reproduction chez la ratte et sur la composition des lipides hépatiques des jeunes. Ann. Nutr. A/im., 30, 67-88.

POTTEAU B., 1976b. Transfert d'acides monomères à structure cyclique dans le lait de la ratte ingérant de I'huile de lin thermopolymérisée. Ann. Nutr. Alim., 30, 89-93.

POTTEAU B., DUBOIS P., RIGAUD J., 1978. Identification et dosage des acides monomères à structure cyclique hydrogénés obtenus à partir d'une huile de lin thermopolymérisée et d'une huile de lin oxydée thermiquement. Ann. Technol. agric., 27, 655-679.

POTTEAU B., GRANDGIRARD A., 1974. Effets toxiques d'une intubation unique de différentes fractions préparées à partir d'huiles chauffées chez le jeune rat. Ann. Biol. anim. Bioch. Biophys., 14, 855-859.

POTTEAU B., GRANDGIRARD A., LHUISSIER M., CAUSERET J., 1977. Recherches récentes sur les effets physiopathologiques d'huiles végétales chauffées. Bibl. Nutr. Diet., N²5, 122-133.

POTTEAU B., LHUISSIER M., LECLERC J., CUSTOT F., MEZONNET R., CLUZAN R., 1970. Recherches sur la composition et les effets physiologiques de l'huile de soja chauffée et de différentes fractions obtenues à partir de cette huile. Rev. fr. Corps gras, 17, 143-153 et 235245.

RAO M. K. G., HEMANS C., PERKINS E. G., 1973. Effect of heated fat upon metabolism of $1-{ }^{14} \mathrm{C}$-acetate in the rat. Lipids, 8, 342-347. 
RAULIN J., 1960. Influence de la configuration stéréochimique des acides gras sur la valeur nutritive des lipides alimentaires. Ann. Nutr. Alim., 14, 201-217.

SAITO M., KANEDA T., 1976. Studies on the relationship between the nutritive value and the structure of polymerized oils. $X$. Structures and toxicity of heat-polymerized oils (en japonais). Yukagaku, 25, 79-86.

SNEDECOR G. W., COCHRAN W. G., 1957. Méthodes statistiques. Association de coordination technique agricole, Paris. $6^{\mathrm{e}}$ éd.

VAN TILBORG H., DE BRUIJN J., GOTTENBOS J. J., KOCH G. K., 1970. Metabolism of orthoalkylbenzenealkanoic acids in rats. J. am. Oil Chem. Soc., 47, 430-437.

VLES R. O., GOTTENBOS J. J., 1972. Long-term effects of feeding butterfat, coconut oil, and hydrogenated or non hydrogenated soyabean oils. I. Eighteen-month experiment in mice. Voeding, 33, 428-433. 